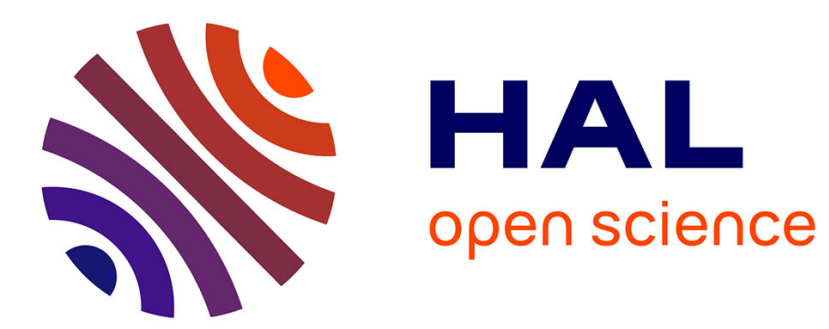

\title{
Ultrasound scattering by forced laminar wakes
}

\author{
Rodrigo H. Hernández Pellicer, Christophe Baudet
}

\section{To cite this version:}

Rodrigo H. Hernández Pellicer, Christophe Baudet. Ultrasound scattering by forced laminar wakes. Vortex Structure and Dynamics, pp.173-189, 2001, 978-3-540-44535-7. 10.1007/3-540-44535-8_10 . hal-00183323

\section{HAL Id: hal-00183323 \\ https://hal.science/hal-00183323}

Submitted on 27 Feb 2020

HAL is a multi-disciplinary open access archive for the deposit and dissemination of scientific research documents, whether they are published or not. The documents may come from teaching and research institutions in France or abroad, or from public or private research centers.
L'archive ouverte pluridisciplinaire HAL, est destinée au dépôt et à la diffusion de documents scientifiques de niveau recherche, publiés ou non, émanant des établissements d'enseignement et de recherche français ou étrangers, des laboratoires publics ou privés. 


\title{
Ultrasound Scattering by Forced Laminar Wakes
}

\author{
Rodrigo H. Hernández ${ }^{1}$ and Christophe Baudet ${ }^{1}$ \\ 1 LEAF-NL, Depto. Ingeniería Mecánica \\ - Universidad de Chile Casilla 2777, Santiago, Chile \\ 2 Laboratoire de Physique (UMR 5672), \\ Ecole Normale Supérieure de Lyon \\ 46 Allée d'Italie 69364 Lyon, France.
}

\section{Introduction}

Most of flows found in nature belong to the class of spatially developing open flows. In this hydrodynamic class, fluid particles enter and leave the flow boundaries of the observation domain continuously. It is the case of boundary layers $[1]$, jets $[3,4]$ and notably wakes [5].

Actually, concepts of absolute and convective instability have proved to be useful to classify the different types of open flows according to their local dynamic behavior. A flow system like the wake, created by the presence of a thin flat plate, fall into the class of noise amplifiers [6,7]. The system become very sensitive to external noise which it is therefore amplified. It is well known that every portion of the wake is convective (stable or unstable); the system presents an extrinsic dynamics, what means that the spatial evolution of the flow is essentially determined, either by the external noise entering the system or by a coherent particular applied forcing. A wake can also display an intrinsic dynamics either by some adequate hydrodynamic resonance or by the onset of localized regions of absolute instability. Vortical structures that are formed become however very insensitive to the incoming external noise and display a very definite oscillation frequency [9].

A renewed interest on wakes comes from the idea of disorder and, perhaps more properly, transition to turbulence. The wake of a thin flat plate does not present spontaneous self-sustained oscillations. Initial disturbances can grow and then saturate but are continuously advected by the mean flow. However its sensitiveness to coherent external forcing makes possible to recover a very rich family of spatially evolving vortical structures. The onset of dynamical vortical structures at large and small scales will determine any further behavior of the wake.

This paper is concerned with the pattern forming structures on the wake of a flat plate under forcing. This choice was made mainly because the laminar velocity profile downstream the plate shows two well defined symmetric sheets of vorticity (of opposite sign). If the plate is submitted to a controlled forcing, one will find its prints in the wake under the form of a spatio-temporal modulation of velocity profiles and thereafter the onset of spatial modes of vorticity. These modes make the modulated wake a benchmark for an ultrasound scattering experiment. 
Scattering of sound waves of high frequency in air by laminar and turbulent vortex flows has been the subject of some recent experimental [10-12] as well as theoretical works [13-15]. The presence of vorticity and the further non linear coupling between the sound wave and the base vortex flow guarantees a coherent sound emission (scattering) which gives considerable information of its spatial structure as well as about its temporal dynamics.

Theoretical works have clearly concluded that the process of sound scattering is well adapted for an acoustic flow diagnostic. In the first Born approximation, the sound scattered pressure (or density) is found to be proportional to the Fourier transform in space and time of the vorticity of the base flow $\boldsymbol{\Omega}(\mathbf{k}, \nu)$, where $\mathbf{k}$ is the wave vector and $\nu$ is the frequency. This constitutes a spectral probe of the vortex flow structure.

Well controlled and coherent vortical structures are obtained if we remain in the category of noise amplifiers. Therefore the flat plate must be very thin, i.e., the thickness-to-length ratio must be small, $e / b \ll 1$. Thus, if the wake is stationary, one can apply an adequate forcing without triggering eigenmodes caused by any instability onset. On the contrary, the system falls into the class of oscillators $[7,16]$ presenting an intrinsic dynamics. Even if fluid particles leave the domain of interest, they remain enough time inside so that infinitesimal disturbances can grow, giving place to the onset of global modes of the system. Comprehensive examples are the flow around a circular $[8,9]$ or square (varying aspect ratio) $[17,16,18]$ cylinders.

At low values of the plate's Reynolds number, $R e_{t}=U_{o} e / \underline{\nu}$, (where $U_{o}, e, \underline{\nu}$ are the free stream mean velocity, plate thickness and kinematic viscosity respectively), and if the boundary layer remain laminar without separation, the near wake remain stationary. Even though two small recirculation regions attached to the trailing edge of the plate always exists [17]. Recirculation regions are unavoidable, even if the cross-stream plate dimension (thickness $e$ ) is small. However the smaller the thickness $e$ the shorter the turnover time will be, so avoiding the onset of instabilities of the wake originated on these recirculating regions.

Modulation of the velocity profile downstream from the plate is performed through a harmonic forcing. A flat plate performing rotary oscillation about the leading edge, creates necessarily a vorticity modulated wake. This is accompanied by the onset of well defined spatial modes of vorticity because the forcing is a priori known.

Forcing consists in making vary the angle of attack of the plate through a periodic oscillation of the plate support (see Figure 1). A very broad range of spatial patterns of vorticity $\boldsymbol{\Omega}(\mathbf{r}, t)$ can be obtained through simple settings of the wave form amplitude $\zeta_{m}$, the uniform upstream velocity $U_{o}$, and the wave form frequency $f_{m}$.

If the oscillation amplitude $\zeta_{m}$ is too high (and therefore the angle of attack $\alpha$ ) there will be strong longitudinal pressure gradients along the plates's faces. Boundary layer separation will be enhanced and further but undesired vortex shedding sets up, perturbing the initially modulated wake pattern [19]. In 


\section{(a)}

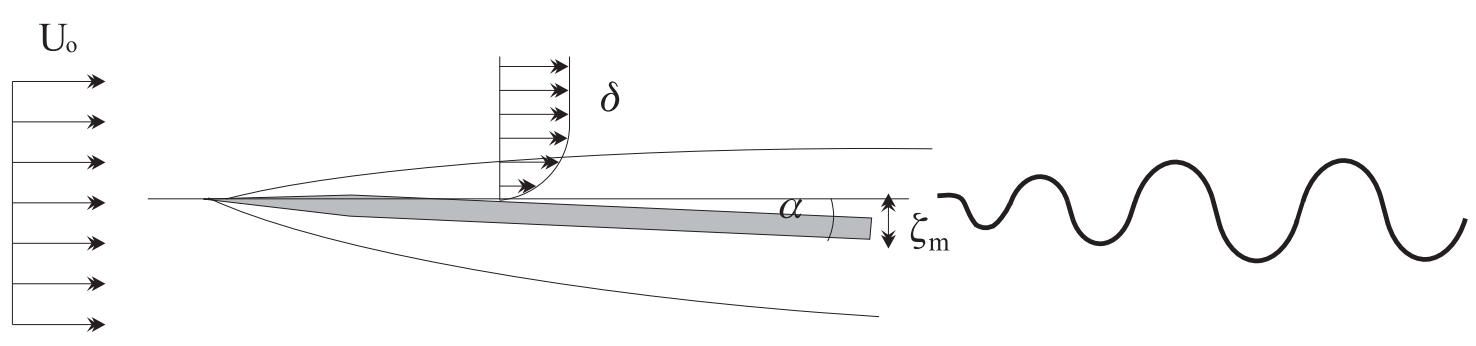

(b)

$\mathrm{U}_{\mathrm{o}}$

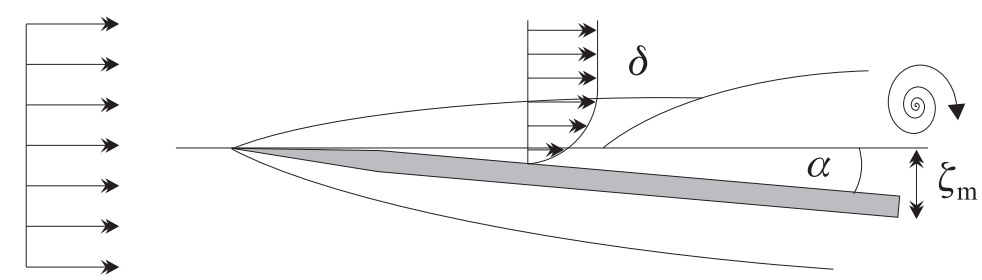

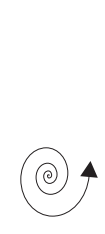

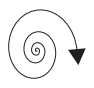

Fig. 1. Expected periodic patterns on a modulated wake through a periodic forced oscillation of the trailing edge of a thin flat plate. (a) Wavy wake for small forcing amplitude $\zeta_{m}$, (b) Large scale vortex shedding for higher forcing amplitude $\zeta_{m}$.

this case, we will get nevertheless a vorticity-modulated wake, but over spatial scales of the order of the length of the plate. An obvious very interesting case but inadequate for an ultrasound scattering experiment in air, where the sound wavelength is too small with respect to those spatial scales. Therefore here the modulation amplitude, $\zeta_{m}$, will be kept of the order of $e$.

This situation is doubly advantageous. First the transverse (cross stream) spatial scale of vorticity is small enough and therefore better adapted to ultrasound sound wavelengths. On the other hand a linear forcing becomes feasible at even higher frequencies.

Even using a very simple way of forcing like here, the wake's spectral dynamics can be very complicated due to non linear interactions. At first, we can expect at least two scenarios as a function of $\zeta_{m}$ and $U_{o}$ according to the symmetry of the vorticity distribution that one gets (Figure 1).

This system presents two characteristic time-scales: one given by the forcing frequency, $f_{m}^{-1}$ and the other by the ratio $b / U_{o}$ both of them defining a characteristic dimensionless parameter $F=f_{m} b / U_{o}$ of the forced wake.

At low forcing frequencies, $f_{m}$ for a $F<1$ condition, we get a slowly varying streamwise sinusoidal wake pattern, where the wave number or spatial mode is given by $2 \pi / \lambda_{\Omega}$ and $\lambda_{\Omega}=U_{o} / f_{m}$ is the resulting vorticity wavelength. At each forcing cycle, fluid particles under the boundary layer have already left the surface of the plate, therefore they 'feel' a non localized spectral forcing (frequency is undefined).

On the contrary, if we increase $f_{m}$ approaching the $F>1$ case, the vorticity distribution becomes more complicated, since fluid particles don't have left the 
plate before a cycle of oscillation takes place. So particles under the boundary layer are now submitted to a harmonic forcing in time. We can now trigger instabilities of the boundary layer itself [2].

In order to remain in the neighborhood of the $F \sim 1$ condition, but working with reasonable high forcing frequencies $f_{m}$, we chose a plate where the width $b$ is small enough so that $F \sim 1$ but greater than $e$ so that we don't loose the class of noise amplifier of the whole system for a wide range of flow velocities $U_{o}$.

\section{Experimental Set Up}

We use a metal (copper, small roughness) thin plate of length $l=20 \mathrm{~cm}$, width $b=4 \mathrm{~cm}$ and thickness $e=1.6 \mathrm{~mm}$. The plate is placed in the test section of an open low turbulence wind tunnel. (maximum free stream velocity $U_{o}=2 \mathrm{~m} / \mathrm{s}$, rate of turbulence $0.05 \%$ ). The leading and trailing edges of the plate are of semi-cylindrical and triangular shape respectively [20].

The plate is maintained in vertical position through two half-axes passing through the geometric center of the front end of the plate (at the middle of the test section). We use ball-bearings supporting axes to perform oscillatory forcing without too much friction. (Figure 2a).

Oscillatory forcing is performed with a B\&K electromagnetic shaker coupled to a rigid aluminum arm system which is fixed on both sides of the plate. The whole system was mounted on a completely independent support, uncoupled from the wind tunnel in order to avoid vibrations associated to fans. Measures of wake velocity profiles were made with a hot wire probe TSI-1210-60 at a distance of $x / e=79.4$ downstream the plate. It can be moved across the wake over $|y / e|<11$ with the help of a computer controlled XY stepper motor system.

Data acquisition, periodic forcing and probe movements were computer controlled using a HP700 series work station and a 4 channel HP3565 spectrum analyzer. A sweep in forcing amplitude as well as in forcing frequency was possible using a HP33120A function generator through the HPIB bus. Forcing waveforms signals were previously amplified by a KEPCO BOP-50-4M amplifier before the B\&K shaker.

The zero attack angle $\alpha=0$ was found iteratively, through a cycle of measures of the symmetry of the velocity profiles downstream the plate. Successive corrections were done through a micrometer screw to change $\alpha$.

\subsection{Sound scattering}

Ultrasound scattering by vortex flows appears as a consequence of non linear coupling between an incident sound wave and a target vorticity distribution. It can be found that the scattered acoustic pressure $p_{s}$ by an arbitrary vorticity field $\boldsymbol{\Omega}(\mathbf{r}, t)$ is directly proportional to the spatio-temporal Fourier transform of vorticity, $\Omega(\mathbf{q}, \nu)$. In three dimensions, using the first Born approximation, $p_{s}$ can be written as [15], 
(a)
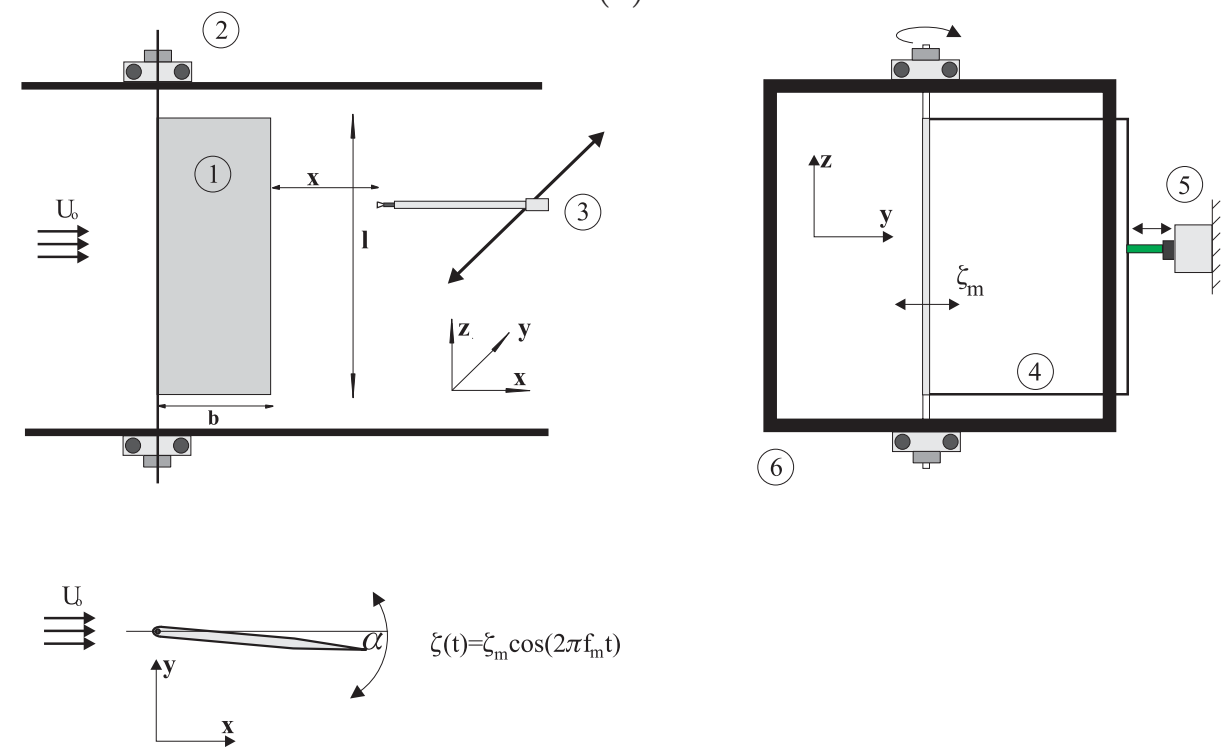

(b)

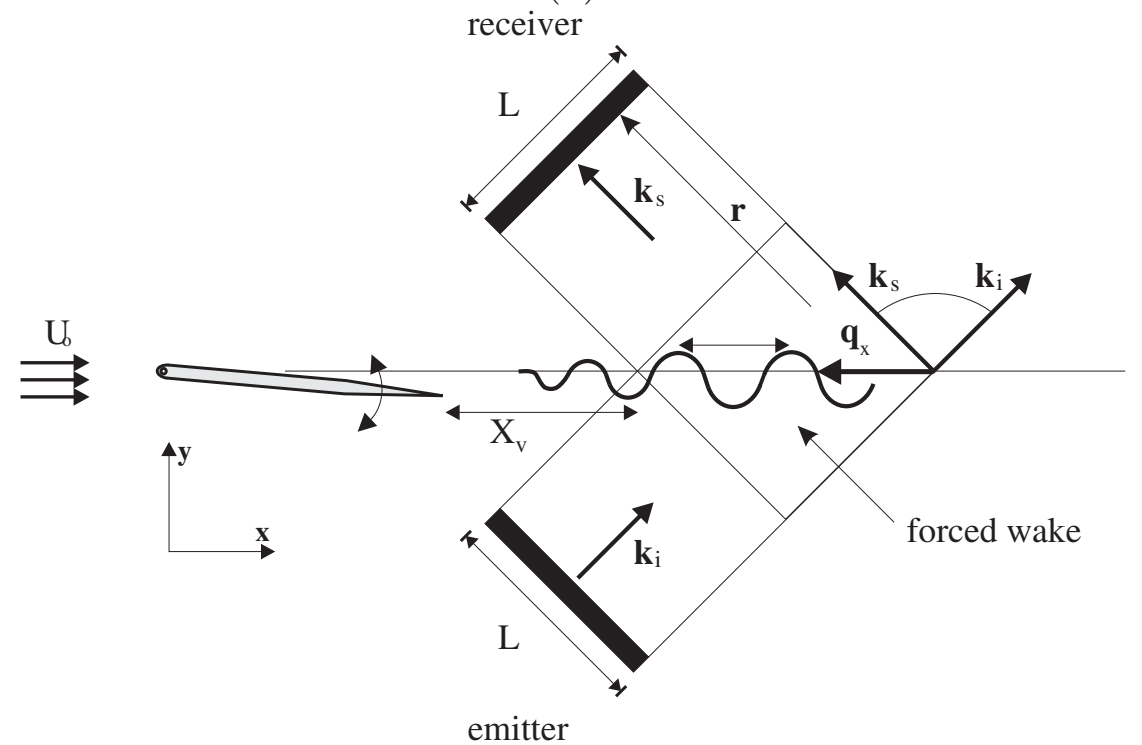

Fig. 2. (a) Experimental Set Up. (1) A vertical metallic flat plate of small roughness. (2) Supporting axes mounted on ball bearings. (3) Hot wire probe scanning the whole test section. (4) Aluminium supporting axes. (5) Shaker. (6) Test section. (b) Sound scattering experimental set up. Square (size $\Lambda=15 \mathrm{~cm}$ ) Sell type ultrasound emitter and receiver under a symmetric configuration. The scattering angle between the incident $\left(k_{i}\right)$ and the scattered $\left(k_{s}\right)$ wave vector is $\theta=30^{\circ}$. The beginning of the scattering volume, $x_{v}$, corresponds to a $x_{v} / b=13$ ratio downstream the plate.

$$
p_{s}(\mathbf{r}, \nu)=p_{o} \frac{i \nu \pi^{2}}{c^{2}|\mathbf{r}|} \mathrm{e}^{i 2 \pi \nu \mathbf{r} / c} \frac{\cos \theta}{1-\cos \theta} \sin \theta \Omega_{z}\left(\mathbf{q}, \nu-\nu_{o}\right)
$$


Where $p_{o}$ is the incident sound pressure, $\theta$ the scattering angle, $\mathbf{r}$ the distance from the target, $c$ the speed of sound and $\nu_{o}$ the incoming sound frequency. The scattering wave vector is defined by $\mathbf{q}=\mathbf{k}_{s}-\mathbf{k}_{i}$, and can be written as $q \sim 4 \pi\left(\nu_{o} / c\right) \sin (\theta / 2) \hat{x}$ in our experimental scattering set up (because $\mathbf{q} \cdot \hat{y}=0$ ).

Sound scattering experiments were performed under the symmetrical configuration shown by figure $2 \mathrm{~b}$ ). Both the sound emitter and receiver are square Sell type transducers of size $\Lambda=16 \mathrm{~cm}$ having a flat response between 5 to $100 \mathrm{kHz}$ within $10 \mathrm{~dB}$. A detailed description can be found in previous work on sound scattering experiments $[10,11]$.

The emitter and receiver (placed outside the test section) are focused toward the wake of the plate (Figure $2 \mathrm{~b}$ ). The incident, $\mathbf{k}_{i}$, and scattered, $\mathbf{k}_{s}$, wave vectors forming an angle $\theta$. With such a geometry we probe the vortex flow at length scales corresponding to the wave vector $\mathbf{q}=\mathbf{k}_{s}-\mathbf{k}_{i}$ whose components are $q_{y}=0$ and $q_{x}=4 \pi\left(\nu_{o} / c\right) \sin (\theta / 2)$. For the actual set-up, considering a constant angle $\theta=30^{\circ}$, the wave vector q can be varied simply by changing the emitter frequency $\nu_{o}$ thus probing different length scales associated to the base vortex flow.

Diffraction effects coming from the limited size of the sound transducers were measured with a transmission diffraction grating of constant step $1.70 \pm 0.01 \mathrm{~cm}$. We found a measured diffraction angle of the order of $3^{\circ}$ at $40 \mathrm{kHz}$.

We will see that the presence of the mean flow introduces a frequency shift on the acoustic signal, a Doppler effect [10], around the incoming frequency $\nu_{o}$. Broadening of scattering peaks is due to diffraction effects which in turn determine our spectral resolution.

A heterodyne detection procedure (demodulation of the received acoustic signal) gives us an analytic signal [21] of low-frequency that can be easily sampled. The phase of this analytic signal is directly related to the Doppler shift. Working at constant angle $\theta$ the theoretical resolution on a single spectral component is given by, $\Delta q=(4 \pi / \Lambda) \cos \theta / 2[11]$.

\section{Results}

\subsection{Hot Wire Wake Measurements}

To know accurately the wake response to different forcing regimes, we performed systematic measurements of wake velocity profiles using thermal anemometry. Scanning the cross stream coordinate with the hot wire probe gave us a complementary picture of the wake dynamics before to proceed with scattering experiments. Note that we use harmonic forcing in time, i.e., $\zeta(t)=\zeta_{m} \cos \left(2 \pi t f_{m}\right)$ where the amplitude $\zeta_{m}$ remains always weak $(\sim e)$. As we mention above, we will create periodic oscillations of the near wake which are advected downstream, forming spatial vorticity modes given approximately by a simple dispersion relation of the form $\lambda_{\Omega} \sim U_{o} / f_{m}$. Remember that if the system remains as a noise amplifier, we can obtain a very rich family of wake's spatial modes.

Once the zero attack angle is obtained, wake velocity recordings without forcing have the typical aspect shown in figure $3 \mathrm{a}$ ). We can see also the cross 
spatial scale of vorticity computed approximately by $\sim \partial u / \partial y$. It is evident that vorticity has two terms $\Omega=\partial u_{y} / \partial x-\partial u_{x} / \partial y$, but the hot wire probe only measures absolute values of velocity. So, we get only an approximate of cross stream velocity gradients. Accurate vorticity measurements are performed in the scattering experiment.

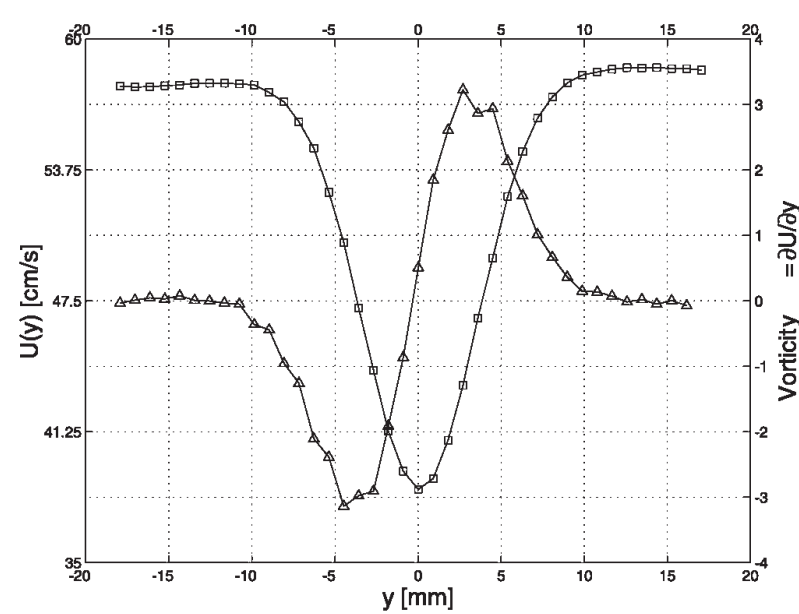

(a)

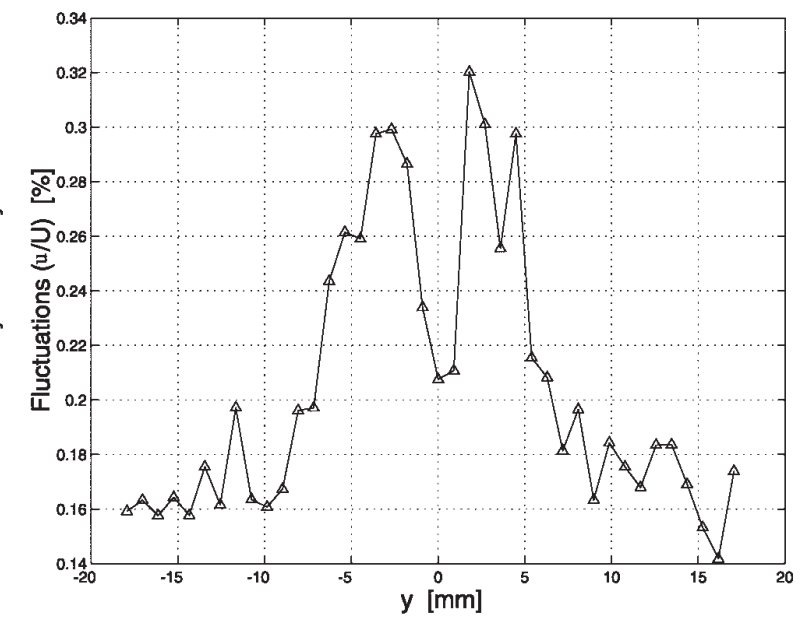

(b)

Fig. 3. Free wake behavior from hot wire measurements at $x / e=79.4$ with $\alpha=0^{\circ}$ and $U_{o}=57 \mathrm{~cm} / \mathrm{s}$. (a) Mean velocity profile $(\sqcup)$. We show also an estimation of the wake's vorticity $(\triangle)$ using first order numerical derivatives. (b) Wake velocity fluctuations.

Even if the wake's velocity fluctuations are weak, $u^{\prime}(y) / \bar{u}$ of the order of $<0.3$ $\%$, two maxima are found precisely where the velocity gradient is maximum (Figure 3b).

This figure gives us an idea of the spatial extent of vorticity, and in particular, of the angle $\beta \sim 2.26^{\circ}$ that determines the (weakly) divergent behavior of the wake. It is an effect of the sharpness of the plate and its width. The Reynolds number based on the plate's width, $R e_{b}$ allow to estimate the boundary layer thickness at the plate's end; $\delta=b / \sqrt{\left(R e_{b}\right)} \sim 1.7 \mathrm{~mm}(\delta / e \sim 1)$. Figure 3a) shows roughly that the cross spatial scale of vorticity is of the order of $3.8 e$.

In general, even at very weak forcing amplitudes, the wake velocity fluctuations are very high. In some cases there is a factor of 10 between them and the natural wake velocity fluctuations (without forcing). On figure 4 we show, as an example, two temporal series of wake velocity under harmonic forcing of constant frequency $f_{m}=10 \mathrm{~Hz}$. We see both the fundamental mode far from the plate $y \sim-8 e$, and the first harmonic close to the plate.

A fundamental difference between a natural and a forced wake is the cross stream increased scale of the later. Figure 5 shows how the wake is enlarged under an harmonic forcing and that this effect is more important at $f_{m}=5 \mathrm{~Hz}$ than $f_{m}=14 \mathrm{~Hz}$. 


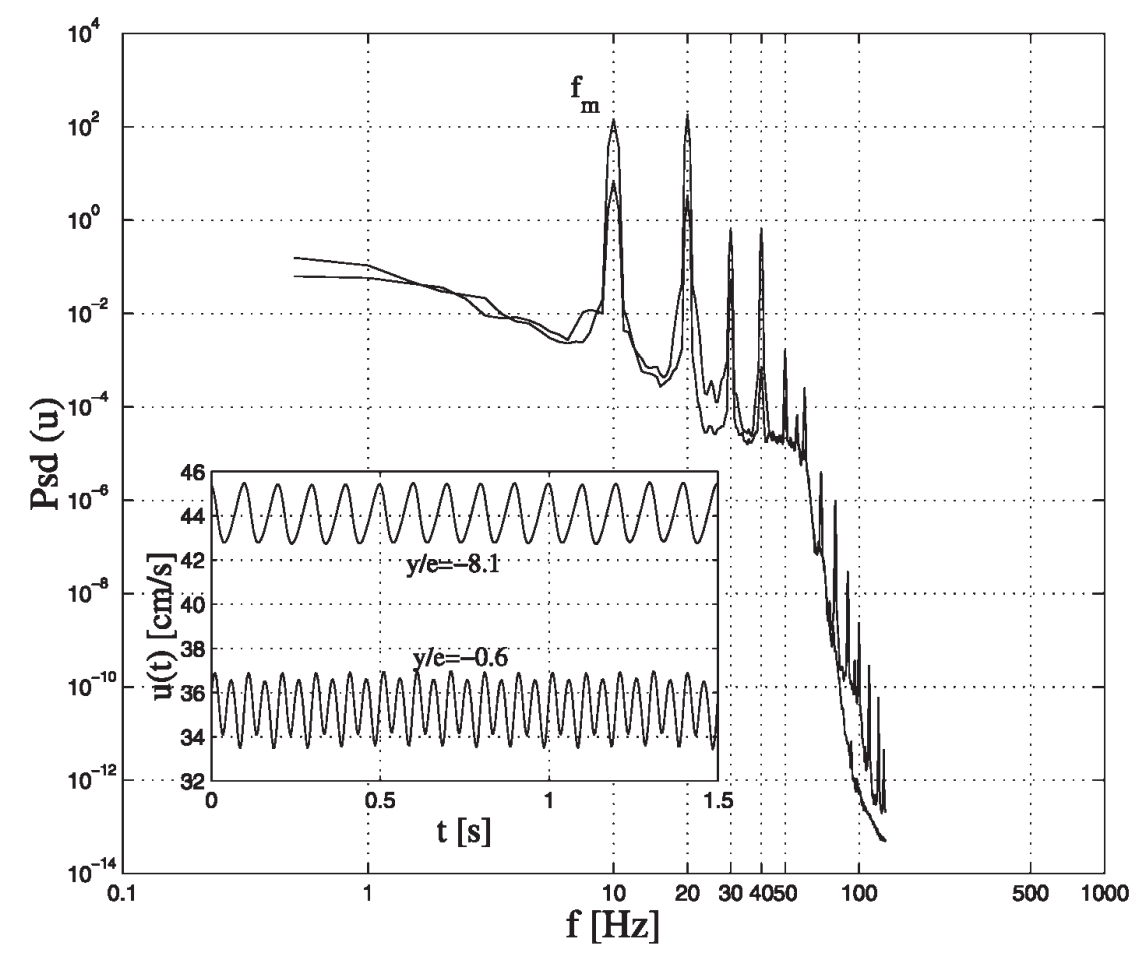

Fig. 4. Forced wake behavior from hot wire measurements. Forcing with amplitude $\zeta_{m}=0.3 \mathrm{~mm}$ at $f_{m}=10 \mathrm{~Hz}$ and Reynolds number $R e_{b}=1185$. Power spectral density computed at two cross stream sites, $y / e=-0.6,-8.1$. The subplot shows typical velocity recordings at those positions.

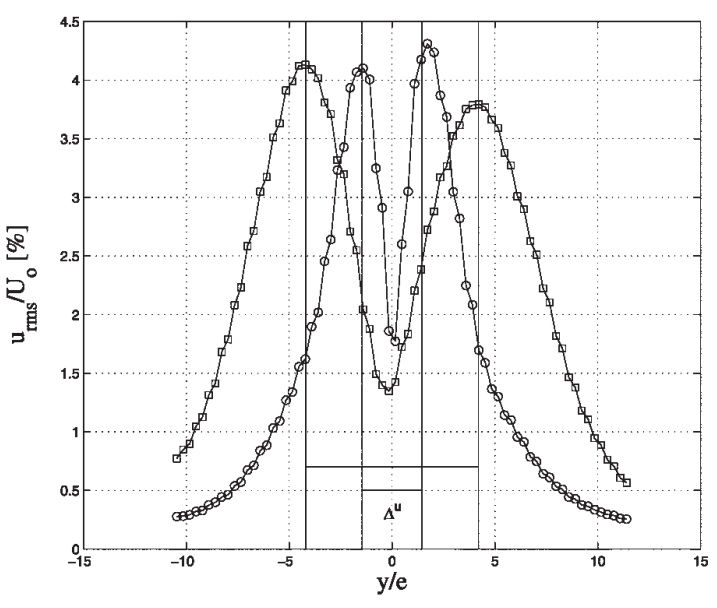

(a)

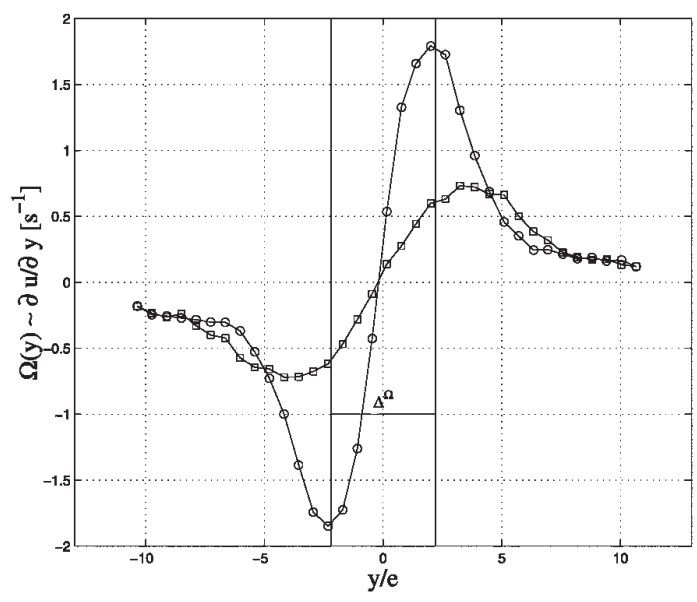

(b)

Fig. 5. Wake forcing at two different frequencies $f_{m}=5$ (०) and $f_{m}=14(\sqcup) \mathrm{Hz}$ with same amplitude $\zeta_{m}=0.3 \mathrm{~mm}$ and Reynolds number $R e_{b}=1185$. (a) The velocity fluctuations $u^{\prime}(y) / U_{o}(x / e=79.4)$ and (b) An estimation of the wake vorticity $\Omega(y)$. We indicate the increased cross stream scale of wake velocity fluctuations $\left(\Delta^{u}\right)$ and vorticity $\left(\Delta^{\Omega}\right)$ with the forcing frequency. 
A comprehensive picture of these effects is found on figure 6 . The shape of mean velocity profiles $u(y)=\overline{u(y, t)}$ and their local fluctuations $u^{\prime}(y)=u_{r m s}$ as a function of the forcing frequency $f_{m}$ (with constant forcing amplitude $\zeta_{m}=$ $0.3 \mathrm{~mm}$ ) is compared with the natural situation (without forcing). We note an increase of both local mean velocity and local fluctuations behind the plate as the forcing frequency decreases. On figure 7 we show the power spectrum of velocity fluctuations at different increasing forcing frequencies. This frequency and its harmonics appears clearly on each plot.

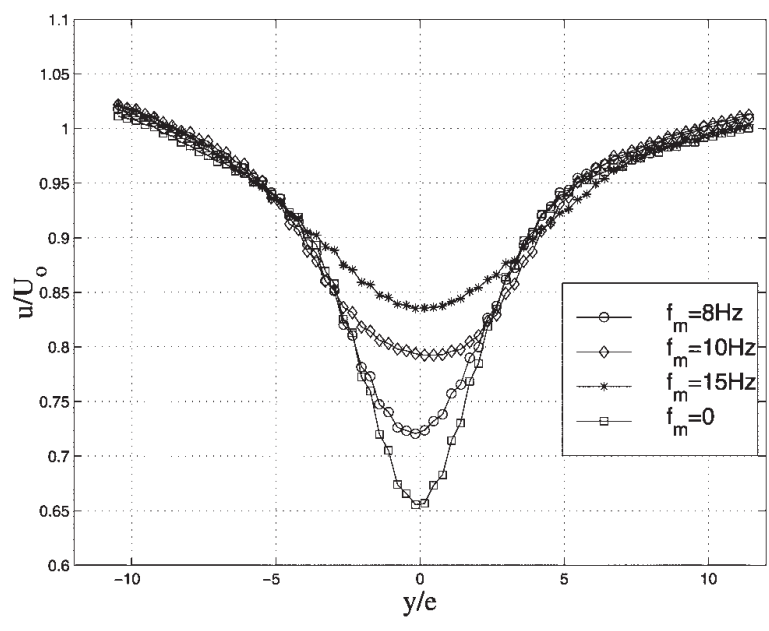

(a)

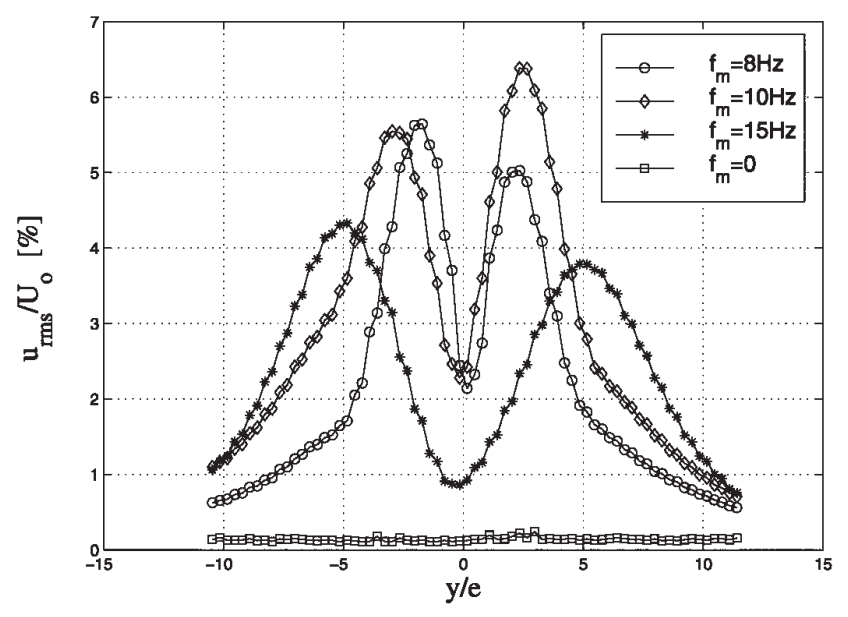

(b)

Fig. 6. Systematic effect of forcing frequency on the wake behavior $(x / e=79.4)$ at constant amplitude $\zeta_{m}=0.3 \mathrm{~mm}$ and Reynolds number $R e_{b}=1185$. (a) Mean velocity profiles $\bar{u} / U_{o}$ and (b) velocity fluctuations $u^{\prime} / U_{o}$. Note that the free wake behavior is indicated by a $\sqcup\left(f_{m}=0 \mathrm{~Hz}\right)$.

\subsection{Sound Scattering and Spectral Wake Dynamics}

As we have shown above, a kind of harmonic forcing like $\zeta(t)=\zeta_{m} \cos \left(2 \pi f_{m} t\right)$ traduces into an oscillatory wake pattern. Any wake modulation traduces into a vorticity modulation at the forcing frequency $f_{m}$.

If we compute the spatial and temporal Fourier transform of the vorticity equation in $2 \mathrm{D}$, neglecting diffusion terms, we obtain a dispersion relation relating frequency and wave number of the form; $2 \pi f=\mathbf{q} \cdot \mathbf{u}$ where $\mathbf{u}$ corresponds to the advection velocity of vorticity. This relationship will explain why we find a Doppler effect associated to scattered pressure signals [11]. We will prove that a scattering peak is found when the scattering wave vector $q$ matches the wave length of the spatial vorticity pattern $\lambda_{\Omega}$ related to the forcing frequency by $\lambda_{\Omega} \sim U_{o} / f_{m}$.

A first agreement with theory (Eq.1) is the fact that the scattering pressure signal, $p_{s}$, shows a Doppler frequency shift $\left(\nu_{o}-f_{m}\right)$, where its sign is given by 


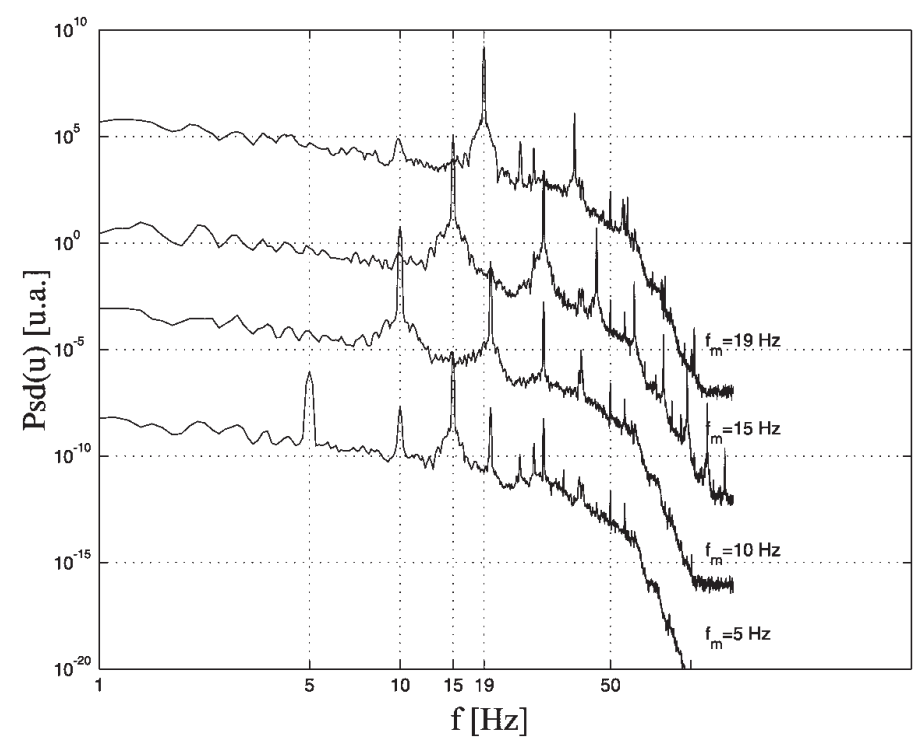

Fig. 7. Power spectra of wake velocity fluctuations at different forcing frequencies. Hot wire measurements performed at $y / e=-8.1, x / e=59.4$ for a constant forcing amplitude $\zeta_{m}=0.3 \mathrm{~mm}$ and Reynolds number of $R e_{b}=10^{3}$.

the sense of the scattering vector $q$. After a heterodyne demodulation, the power spectrum of the scattered pressure signal is centered on $\nu_{o}$ and one measures with good accuracy $(d \nu=50 \mathrm{mHz})$ the spectral line associated to $p_{s}$, as shown by the subplot of figure 8 . The scattering peak appears exactly at $\nu-\nu_{o}=f_{m}$ as predicts the equation 1.

If we change the incoming sound frequency $\nu_{o}$, for a constant scattering angle $\theta / 2$, we are exploring a wide band of spectral components of the wake pattern. Remember that the scattering vector is given by $q=4 \pi\left(\nu_{o} / c\right) \sin (\theta / 2)$.

After a frequency sweep of the incoming sound frequency, $\nu_{o}$, we record the evolution of the Doppler spectral line at $f_{m}$ as a function of the scattering vector $q$. We found, as expected, a resonant behavior for the scattering amplitude $p_{s}$ as shown in figure 8. A resonant scattering vector $q_{r}$ associated to a particular sound frequency, corresponds to the fundamental wave vector of the modulated wake pattern under forcing, as given by the relationship $2 \pi / \lambda_{\Omega}$. The second peak in figure 8 (indicated as $q_{r}^{\Lambda}$ ), originates by diffraction effects due to the finite size window of the sound emitter and receiver. The spectral window is theoretically given by a $\sin _{c}$ profile for square windows. Note that the relative position of the peak $q_{r}^{\Lambda}-q_{r}$ does not change with the mean wake velocity as it was already proved in other experiments [11].

For a constant forcing frequency $f_{m}$ and varying upstream flow velocity $U_{o}$, the spectral wake components will change, obeying to the simple relationship, $\lambda_{\Omega} \sim U_{o} / f_{m}$. If we consider that the sound wave is modulated by the vorticity pattern, the corresponding scattering spectral line obtained at different flow velocities must not change, even though the resonant wave vector $q_{r}$ must be different. This is confirmed on figure 9. The Doppler peak on the power spectrum (Figure 9a) is independent of the flow velocity. However the corresponding 


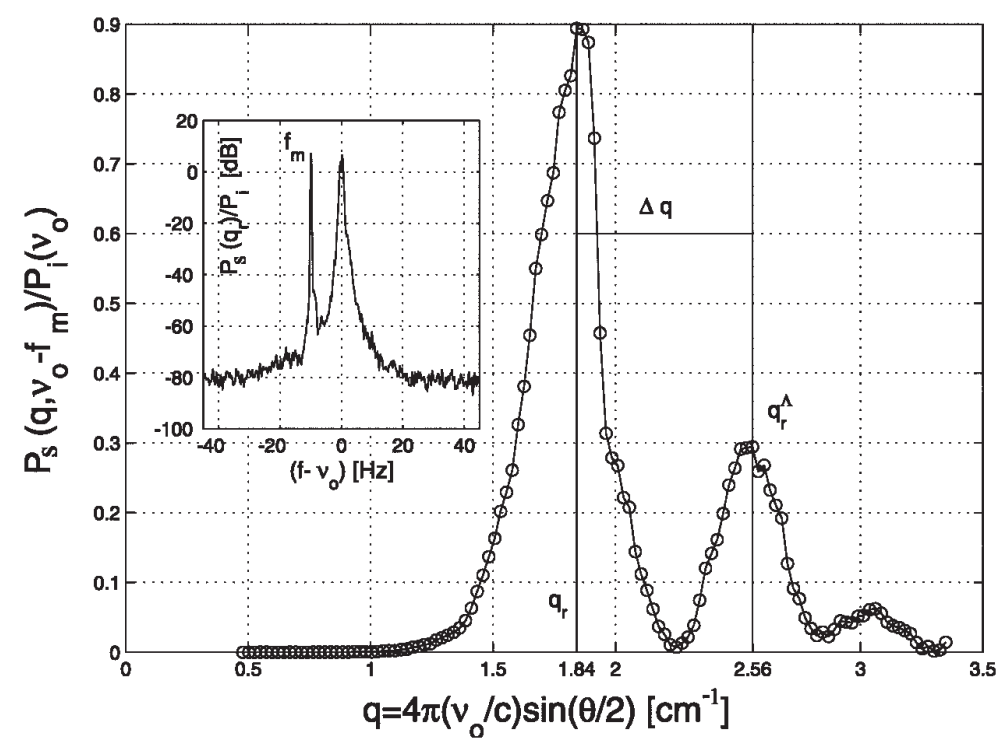

Fig. 8. Ultrasound scattering by the forced wake (forcing amplitude, frequency and Reynolds number are: $\zeta_{m}=0.3, \mathrm{~mm}, f_{m}=10 \mathrm{~Hz}, R e_{b}=10^{3}$ respectively). We display the normalized amplitude of the scattered pressure as a function of the scattering wave vector $q$. The spatial resonance is obtained at $q=q_{r}\left(\nu_{o}=19.25 \mathrm{kHz}\right)$. The subplot shows the corresponding power spectrum of scattered pressure, where a Doppler peak is found at $f-\nu_{o}=f_{m}$.

resonant scattering vectors occur for very different wake pattern wave lengths (Figure 9b).

So we confirm the validity of the dispersion relation. The scalar product $\mathbf{q} \cdot \mathbf{u}$ is exactly conserved using the resonant scattering wave vectors. This picture allow us to understand the sound scattering mechanism, where the incoming sound wave is linearly modulated in frequency, which explains the Doppler shift on scattered pressure signals. This is the reason why we use a heterodyne detection technique, largely used in the radio frequency domain. The carrier wave at $\nu_{o}$ is modulated at $f_{m}$ which corresponds to the scattered pressure by flow vorticity.

If we take a look at the scattering set up (Figure 2b), we see that scattering pressure comes from the volume defined by the intersected acoustic beams (incident and scattered beams) downstream the plate. Therefore the advection velocity for the vorticity field is not exactly the free stream velocity $U_{o}$. An effort was made to determine a characteristic advection velocity from the velocity profiles measured downstream the plate with the hot wire probe.

The advection velocity is simply defined here as the spatial average (in the cross stream direction) of $u(y),\langle u\rangle$,

$$
\langle u\rangle=\frac{1}{Y} \int_{-Y / 2}^{Y / 2} u(y) d y
$$

where $Y$ is the characteristic scale of wake velocity profiles at the beginning of the scattering volume. We can see on figure 10b) that it is a very reasonable definition of advection velocity, because if we compute the dimensionless number 


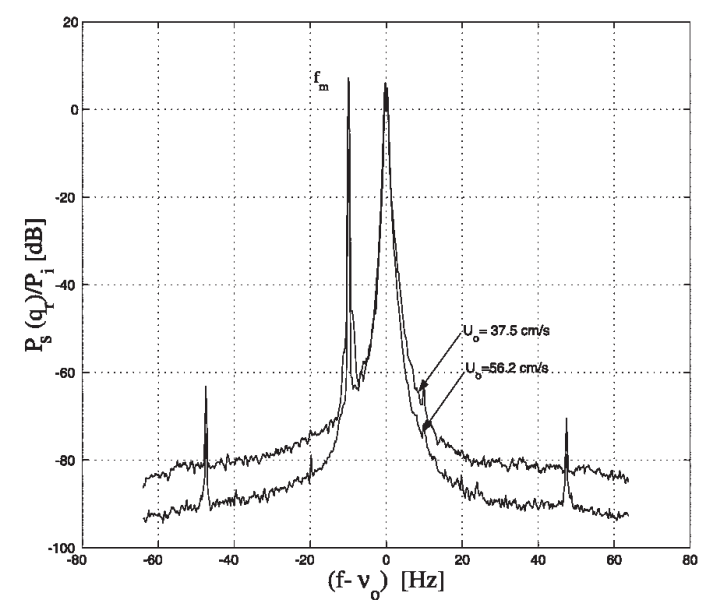

(a)

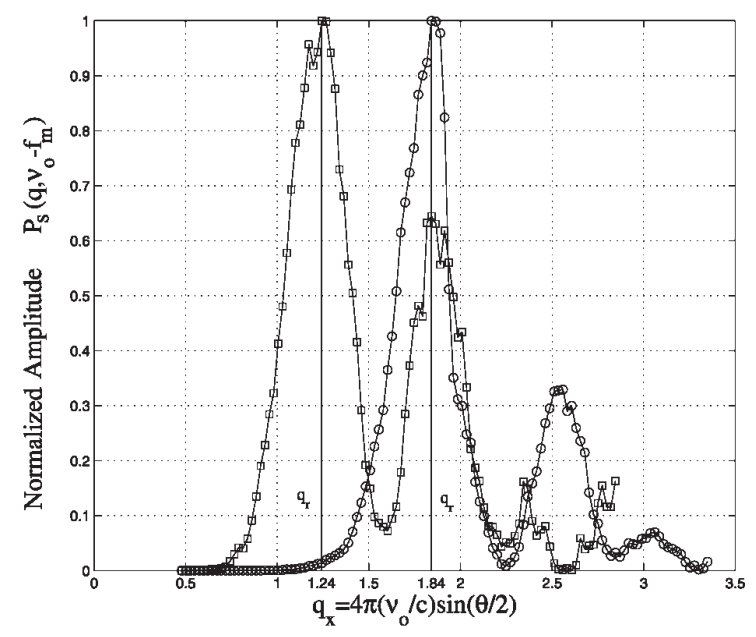

(b)

Fig. 9. Ultrasound scattering by the forced wake at two different free stream velocities $R e_{b}=10^{3}(\sqcup), R e_{b}=1.5 \times 10^{3}(\circ)$, where the forcing amplitude and frequency are: $\zeta_{m}=0.3, \mathrm{~mm}$ and $f_{m}=10 \mathrm{~Hz}$, respectively. (a) The power spectra of scattered pressure display the same Doppler peak at the forcing frequency $f_{m}$. (b) A sweep of the scattering wave vector $q=4 \pi\left(\nu_{o} / c\right) \sin (\theta / 2)$ shows that two clearly different spatial resonances are found at very definite length scales.

$F$ using this velocity $F=f_{m} b /\langle u\rangle$, we found that the resonant wave length $\lambda_{r} / b$ evolves linearly with $F$ as one should expect. Moreover, we found that at $F=1$ both $\lambda_{r} / b \sim 1$ and the resonant scattered pressure amplitude is maximum.

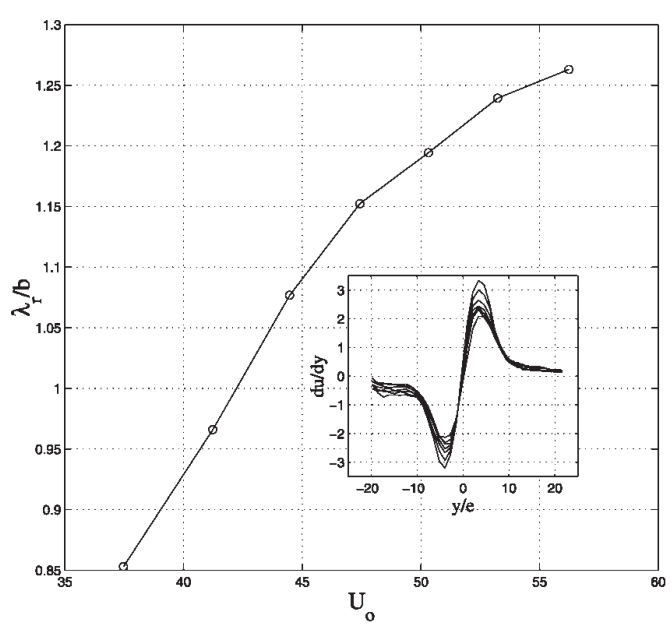

(a)

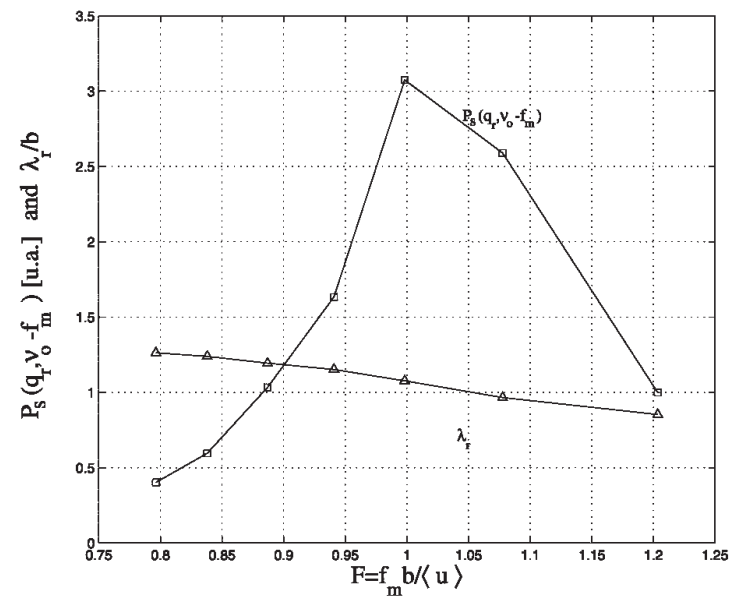

(b)

Fig. 10. (a) Evolution of the normalized resonant wavelength $\lambda_{r}=2 \pi / q_{r}$ with the free stream velocity $U_{o}$ for a forced wake $\left(\zeta_{m}=0.3 \mathrm{~mm}, f_{m}=10 \mathrm{~Hz}\right)$. (b) Evolution of the normalized resonant scattered pressure amplitudes $(\sqcup)$ and resonant wavelengths $(\triangle)$ with the dimensionless parameter $F=f_{m} b /\langle u\rangle$. 
In the vicinity of $F=1$ the path of fluid particles is exactly a half of the plate's width, $b / 2$ on every half forcing period, so the spatial wake pattern has a wave length of order $b^{-1}$. At this scale the associated vorticity of that mode reaches a maximum, i.e., a vorticity-resonance as a function of the forcing frequency. This behavior was unexpected. But it can be explained as follows: Fluid particles inside the boundary layer whose path is exactly $b / 2$ acquire a complete momentum transfer given by the half-forcing cycle, they pick up an increased cross velocity and so increase the wake velocity gradient and therefore vorticity.

At very weak upstream velocities, $U_{o}$, the wake can be considered as linear. But as $U_{o}$ is increased we expect the wake to become non linear. Two effects can be attributed to non linear terms. First, the linear growth of natural as well as artificial instabilities is actually limited or saturated by non linear terms, and second the production of higher harmonics which is the richness of a non linear oscillator system.

Measurements of a non linear wake with hot wire probes make non sense because of the intrinsic non linear character of the hot wire itself (production of harmonics of a pure sinusoidal signal). Therefore it is a well controlled scattering experiment which shed light on such a non linear behavior, due to the fundamental linear relationship between scattering pressure and vorticity (See equation 1).

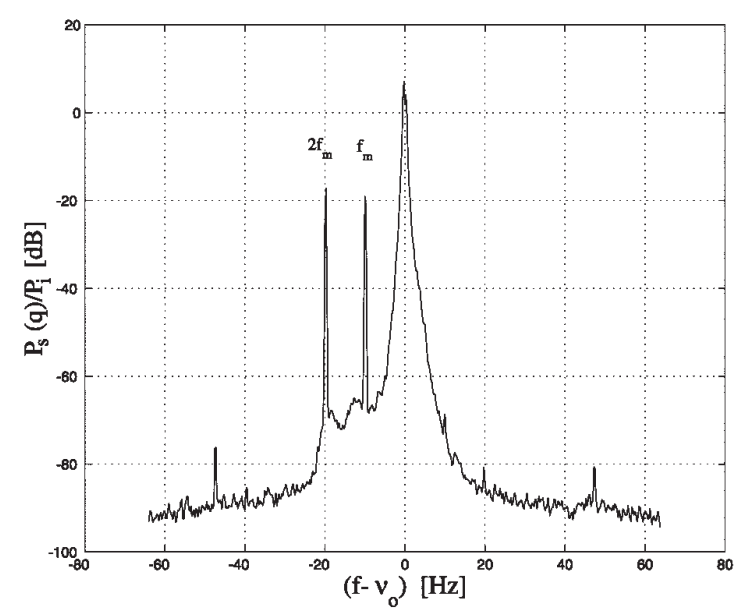

(a)

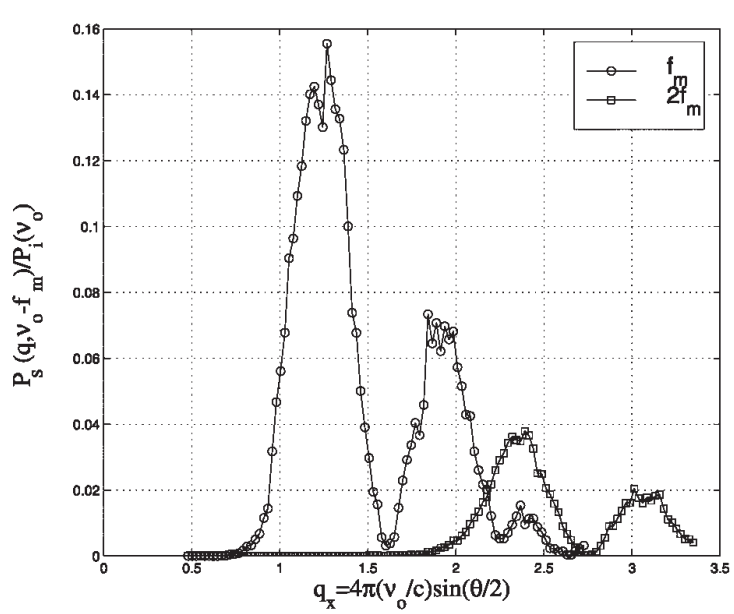

(b)

Fig. 11. Example of higher harmonics production by a non linear wake. Forcing amplitude, frequency and Reynolds number are: $\zeta_{m}=0.3, \mathrm{~mm}, f_{m}=10 \mathrm{~Hz}, R_{e}=1.4 \times 10^{3}$ respectively. (a) Power spectrum of scattered pressure showing two peaks associated to the fundamental $\left(f_{m}\right)$ and first harmonic $\left(2 f_{m}\right)$. (b) Evolution of the peak amplitude of the fundamental $(\circ)$ and first harmonic $(\sqcup)$ as a function of the scattering wave vector $q=4 \pi\left(\nu_{o} / c\right) \sin (\theta / 2)$.

Figures 11 and 12 show such a non linear process. At constant forcing frequency $f_{m}=10 \mathrm{~Hz}$, we increase the upstream velocity or the Reynolds number 


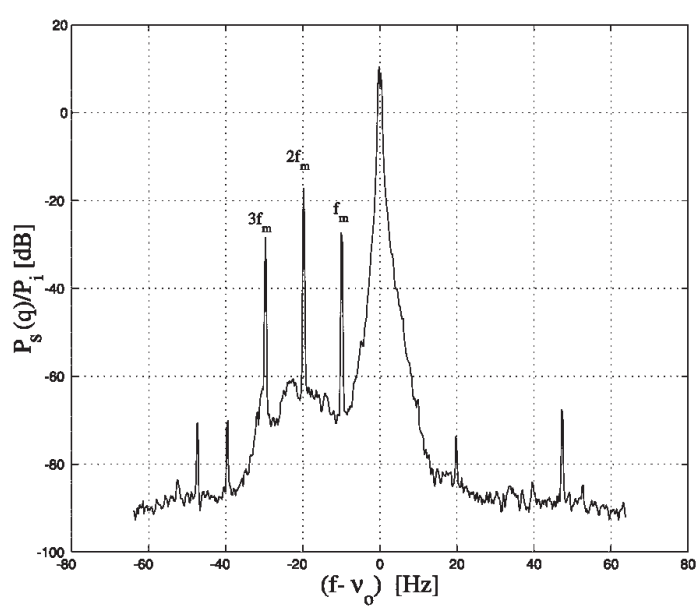

(a)

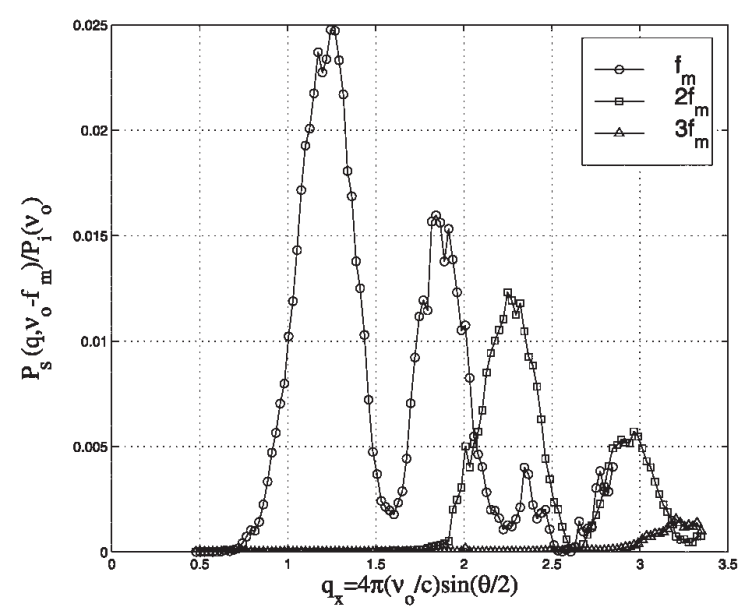

(b)

Fig. 12. Another example of higher harmonics production by a non linear wake. Forcing amplitude, frequency and Reynolds number are: $\zeta_{m}=0.3, \mathrm{~mm}, f_{m}=10 \mathrm{~Hz}, R e_{b}=$ $1.5 \times 10^{3}$ respectively. (a) Power spectrum of scattered pressure showing three Doppler peaks associated to the fundamental, first and second harmonics. (b) Spatial scales associated to the fundamental $(\circ)$, first $(\sqcup)$ and second harmonics $(\triangle)$.

$R e_{b}$. The power spectra in figures 11a) and 12a) show clearly the onset of the first and second harmonics $2 f_{m}$ and $3 f_{m}$ respectively.

The amplitude of the fundamental, first and second harmonics was obtained as before, with a frequency sweep of the incoming sound wave ( See figures 11b and $12 \mathrm{~b}$ ), to resolve accurately each spatial mode.

As we see we are able to resolve the complete temporal and spatial dynamics of a non linear oscillating wake. With this result in mind we pursued a more ambitious forcing. At constant upstream velocity, the forcing wave form is the sum of two waves of incommensurate frequencies.

$$
\zeta(t)=\zeta_{m}\left(\mathrm{e}^{i 2 \pi f_{m}^{1}}+\mathrm{e}^{i 2 \pi f_{m}^{2}}\right)
$$

We digitally synthesized the sum of two pure sinusoidal waves at frequencies of $f_{m}^{1}=8$ and $f_{m}^{2}=10 \mathrm{~Hz}$ with the same amplitude $\zeta_{m}$ recorded on the volatile state memory of the function generator. This forcing serves also to inspect non linearities of the wake. We should find spectral lines at frequencies $\delta f_{m}=f_{m}^{1} \pm f_{m}^{2}$ and successive combinations of the original waves with $\delta f_{m}$.

On figure 13 we see that the power spectrum of scattered pressure at $R e_{b}=$ $10^{3}$ shows four individual spectral lines (peaks) at the following frequencies: $-6,-8,-10,-12 \mathrm{~Hz}$. They are found at the negative frequency axis because of the sign of the corresponding Doppler effect $(\mathbf{q} \cdot \mathbf{u})$. The spectral line at $\delta f_{m}=2$ $\mathrm{Hz}$ can not be seen due to the broadening of the spectral line of the incoming sound wave. However exact combinations between the original waves and the $\delta f_{m}$ wave are demonstrated by the peaks at 6 and $12 \mathrm{~Hz}$. 
Again a sweep in wave number $q$ allow us to find the corresponding spatial modes associated to each peak in the power spectrum. We verify that at each spectral line corresponds a resonant spatial wave vector as indicated on figure $13 \mathrm{~b}), q_{r 1}, q_{r 2}, q_{r 3}, q_{r 4}$ On the subplot of figure 13a) we show the experimental dispersion relation $\omega(q)$ for those vorticity modes.

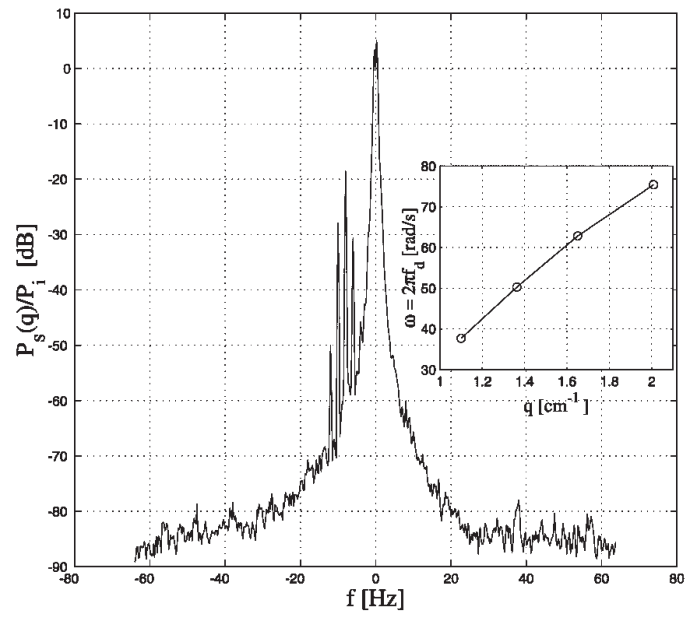

(a)

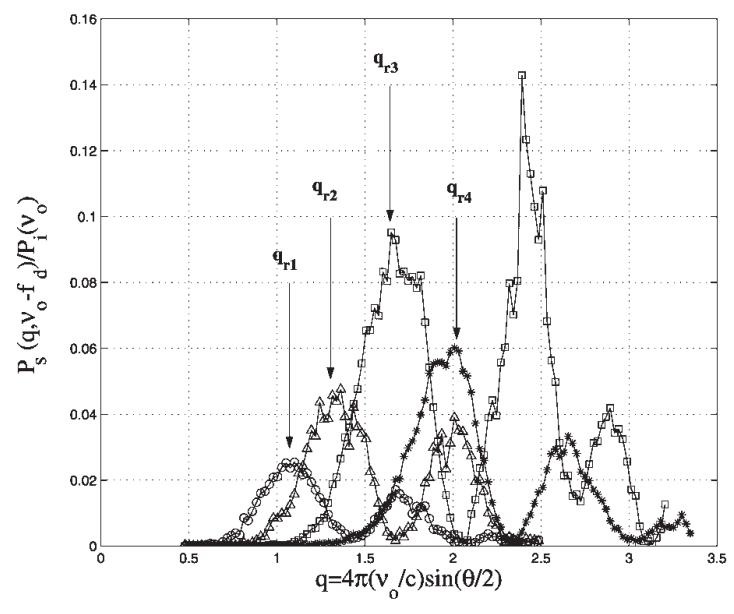

(b)

Fig. 13. Wake modulation at two forcing frequencies $f_{m}^{1}=8, f_{m}^{2}=10 \mathrm{~Hz} .\left(\zeta_{m}=\right.$ $0.3 \mathrm{~mm}, R e_{b}=10^{3}$ ). (a) Power spectrum of scattered pressure showing four Doppler peaks (modes). The subplot showing their dispersion relation $\omega(q)$. (b) Spatial resonances at each Doppler peak, showing the presence of very different spatial modes in the wake. Doppler shifts are $-6 \mathrm{~Hz}(\circ),-8 \mathrm{~Hz}(\triangle),-10 \mathrm{~Hz}(\sqcup)$ et $-12 \mathrm{~Hz}(*)$.

It is evident that if increase the number of forcing modes we should speak of wave packets. The propagating properties of the wake can then be investigated. This experiment immediately suggests that one can study the evolution of wave packets introduced into a laminar wake. We can then follow accurately their evolution in time using ultrasound scattering and try to determine possible energy transfers between different modes. A work of this kind will permit to better understand actual ideas at the origin of energy transfers in turbulent open flows. The mechanical energy being injected at integral length scales is then transferred toward the smaller scales trough non linear coupling, being finally dissipated as heat by viscosity [10].

\section{Conclusion}

The objective of this experimental work was to study the dynamical behavior of a laminar wake under a linear harmonic forcing. Forcing was introduced by small amplitude rotary oscillations of the trailing edge of a flat plate. 
A throughout investigation of the wake behavior and vorticity modulation was accomplished using classical hot wire anemometry and ultrasound scattering methods respectively.

We confirmed the scattering mechanism of sound waves by a target of vorticity. We put forward and confirmed the presence of a Doppler effect in agreement with recent theoretical findings, in particular that the Doppler shift is accurately described by the scalar product $\mathbf{q} \cdot \mathbf{u}$.

Important findings on shedding vorticity were put forward using sound scattering. In particular, the resonant behavior of vorticity with the forcing frequency.

The fundamental difference between this experiment and those of sound scattering by von Kármán vortices [11] is that here $\lambda_{\Omega}$ and $R e$ are not coupled parameters, since we can vary $\lambda_{\Omega}$ independently of the free stream velocity and to probe a wide band of spectral modes associated to the wake pattern.

Experiments on mixed harmonic forcing (the sum of two harmonic waves) can be viewed as an introduction to the propagation of wave packets through laminar wakes. It is the spatial resolution of the spectral measurements, in the limit of diffraction effects, that must permit to work with a vorticity distribution including several spectral modes.

\section{Acknowledgment}

We are grateful to S.Fauve for very fruitful discussions. R.H.Hernández acknowledges support from ECOS (action C94E02)

\section{References}

1. H. Schlichting, Boundary Layer Theory, McGraw Hill, New York, 1968.

2. J. J. Healey, A new boundary layer resonance enhanced by wave modulation: theory and experiment, J. Fluid Mech., 304, 231, 1995.

3. A. Michalke, Survey on jet instability theory, Prog. Aerospace Sci., 21, 159, 1984.

4. B. L. Smith and A. Glezer, The formation and evolution of synthetic jets, Phys. Fluids, 10, (9), 2281, 1998.

5. M. Schumm, E. Berger and P. A. Monkewitz, Self-excited oscillations in the wake of two-dimensional bluff bodies and their control, J.Fluid Mech., 271, 17, 1994.

6. A. Couairon and J. M. Chomaz, Global instability in fully nonlinear systems, Phys.Rev.Lett, 77, 4015, 1996.

7. P. Huerre and P. Monkewitz, Local and Global instabilities in spatially developing flows, Annu.Rev.Fluid Mech., 22, 473, 1990.

8. C. Mathis, M. Provansal and L. Boyer, The Bénard-von Kármán instability: an experimental study near the threshold, J.Physique Lett., 45, L-483, 1984.

9. M. Provansal, C. Mathis and L. Boyer, Bénard-von Kármán instability: transient and forced regimes, J.Fluid Mech., 182, 1, 1987.

10. C. Baudet, S. Ciliberto and J.-F. Pinton, Spectral analysis of the von Kármán flow using ultrasound scattering, Phys.Rev.Lett., 67, (2), 193, 1991.

11. J.-F. Pinton and C. Baudet, Measurements of vorticity using ultrasound scattering, in Turbulence in spatially extended systems, Nova Science Publishers, 1993. 
12. J.-F. Pinton, C. Laroche, S. Fauve and C. Baudet, Ultrasound scattering by buoyancy driven flows, J.Phys. II France, 3, 767, 1993.

13. A. L. Fabrikant, Sound scattering by vortex flows, Sov.Phys.Acoust., 29, (2), 152, 1983.

14. L. M. Lyamshev and A. T. Skvortsov, Sound scattering by a vortex soliton in axisymmetrical shear flow, Sov.Phys.Acoust. 35, (3), 279, 1989.

15. F. Lund and C. Rojas, Ultrasound as a probe of turbulence, Physica D, 37, 508, 1989.

16. K.Hannemann and H.Oertel Jr., Numerical simulation of the absolutely and convectively unstable wake, J.Fluid Mech., 199, 55, 1989.

17. H. Oertel Jr., Wakes behind blunt bodies, Annu.Rev.Fluid Mech., 22, 539, 1990.

18. A. Okajima, Strouhal numbers of rectangular cylinders, J.Fluid Mech., 123, 379, 1982.

19. S. Taneda, Visual obserbations of the flow past a circular cylinder performing a rotatory oscillation, Journal of the Physical Society of Japan, 45, (3), 1038, 1978.

20. S. Candel, Mécanique des fluides, Dunod, Paris, 1990.

21. A. Papoulis, Probability, Random Variables and Stochastic Processes, McGraw Hill, New York, 1965. 